\title{
Challenges in the noninvasive detection of body composition using near-infrared spectroscopy
}

\author{
Wenliang Chen*,†, Hao Jia*, Chenli Li* \\ and Kexin $\mathrm{Xu}^{*, *}$ \\ *State Key Laboratory of Precision Measuring Technology \\ and Instruments, Tianjin University, Tianjin 300072, P. R. China \\ ${ }^{\dagger}$ Key Laboratory of Micro-Optical-Electro-Mechanical System Technology \\ Tianjin University, Ministry of Education, Tianjin 300072, P. R. China \\ tkexin@tju.edu.cn
}

Received 18 July 2013

Accepted 3 November 2013

Published 19 December 2013

\begin{abstract}
Noninvasive detection of body composition plays a significant role in the improvement of life quality and reduction in complications of the patients, and the near-infrared (NIR) spectroscopy, with the advantages of painlessness and convenience, is considered as the most promising tool for the online noninvasive monitoring of body composition. However, quite different from other fields of online detection using NIR spectroscopy, such as food safety and environment monitoring, noninvasive detection of body composition demands higher precision of the instruments as well as more rigorousness of measurement conditions. Therefore, new challenges emerge when NIR spectroscopy is applied to the noninvasive detection of body composition, which, in this paper, are first concluded from the aspects of measurement methods, measurement conditions, instrument precision, multicomponent influence, individual difference and novel weak-signal extraction method based on our previous research in the cutting-edge field of NIR noninvasive blood glucose detection. Moreover, novel ideas and approaches of our group to solve these problems are introduced, which may provide evidence for the future development of noninvasive blood glucose detection, and further contribute to the noninvasive detection of other body compositions using NIR spectroscopy.
\end{abstract}

Keywords: Near-infrared spectroscopy; noninvasive detection; body composition; blood glucose.

\section{Introduction}

Noninvasive body composition detection plays a significant role in the improvement of life quality and the reduction of complications of the patients. Taking diabetes as an example, there are about
350 million patients all over the world. ${ }^{1}$ In China, it accounts $9.7 \%$ of the total adults, which is $\sim 90$ million, $3 / 4$ of which are suffering from complications such as atherosclerosis, cardiovascular diseases and diabetic nephropathy. ${ }^{2}$ Nowadays, blood

This is an Open Access article published by World Scientific Publishing Company. It is distributed under the terms of the Creative Commons Attribution 3.0 (CC-BY) License. Further distribution of this work is permitted, provided the original work is properly cited. 


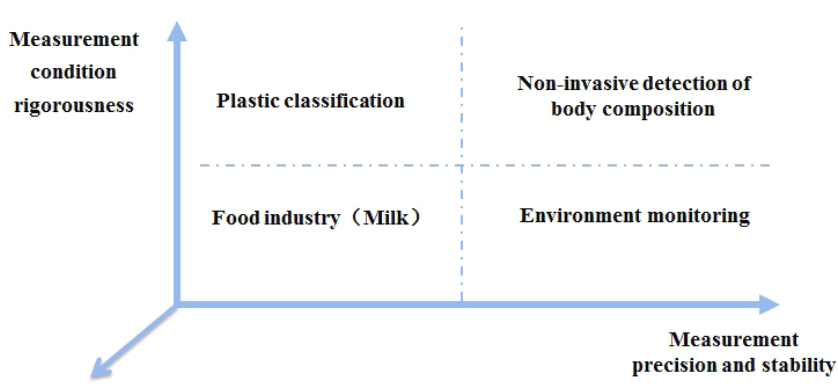

Fig. 1. Requirements in different fields of NIR noninvasive detection.

glucose detection still depends largely on the frequent finger prick sampling, which usually bring pain and fear to the patients despite minimum invasion. $^{3}$ Therefore, near-unfrared (NIR) spectroscopy, with advantages of painlessness and convenience, is considered as the most promising tool to achieve noninvasive detection of body composition. ${ }^{4}$

However, compared with other noninvasive detection fields of using NIR spectroscopy, such as food safety and environment monitoring, the noninvasive detection of body composition requires higher precision and stability of the instruments as well as more rigorous measurement conditions, as indicated in Fig. 1.

Therefore, new challenges emerge when NIR spectroscopy is applied to the noninvasive detection of body composition, and the precision has not yet been clinically realized due to the following reasons. First, the interesting body composition, for example, the blood glucose, is usually trace in amount, so the useful signal in the NIR spectra is quite weak to detect. Second, the human body is commonly defined as complex scattering medium in the biomedical photonics. Concentrations of other components also vary from time to time because of the metabolism (which is called background shift by our group). Other factors, like the fluctuation of body temperature, can also bring great interference to the measurement. All these influences inevitably result in measurement error of the interesting component. Third, individual difference limits the weak-signal extraction method. When differences exist in the optical parameters of the tissue, they will cause changes in the NIR spectra, but the interesting component remains stable. Besides, instrument precision is also one of the major influencing factors in the noninvasive detection of body composition. ${ }^{5}$

In this paper, based on our previous research in the cutting-edge field of NIR noninvasive blood glucose detection, the new challenges in the noninvasive detection of body composition using NIR spectroscopy are first concluded from the aspects of measurement methods, measurement conditions, instrument precision, multi-component influence, individual difference and novel weak-signal extraction method. Moreover, novel ideas and approaches proposed by our group to solve these problems are introduced, which may provide evidence for the future development of noninvasive blood glucose detection and further contribute to the noninvasive detection of other body compositions using NIR spectroscopy.

\section{New challenges in the Noninvasive Detection of Human Body Compositions Using NIR Spectroscopy}

\subsection{Measurement methods}

The NIR spectroscopy has been adopted with maturity in the areas of food safety and environment monitoring, and many online detections have been successfully developed, such as composition detections of grain crops, ${ }^{6}$ tobacco ${ }^{7}$ and identification of fruit maturity. ${ }^{8}$ Also, the flue gas, such as escaping $\mathrm{NH}_{3}$ monitoring has also achieved high precision using NIR TDLAS. ${ }^{9}$ However, the NIR noninvasive detection of body composition is not easily realized, since the Lambert-Beer's law cannot be fully satisfied as the human body is a complex scattering medium, the concentration of interesting component is usually trace in amount, and the optical path length is unknown. Therefore, the NIR noninvasive detection of body composition calls for development of novel measurement methods. ${ }^{10}$

First attempt to the body composition detection using optical method was taken by Kaiser ${ }^{11}$ and Jobsis $^{12}$ in the 1970s. In 1987, Dähne et al. first proposed the noninvasive measurement of blood glucose using NIR absorption spectroscopy. ${ }^{13}$ More and more attention were then paid to the noninvasive blood glucose detection from 1990 to 1998 by either transmission or scattering methods,${ }^{14}$ while novel methods based on the diffuse-reflectance method bloomed, such as OCT ${ }^{15}$ and metabolic heat measurement. ${ }^{16}$ Especially since 2006, the focus was emphasized again on the method innovation. For example, OrSense Company of Israel tried to realize dynamic and precise measurement of blood glucose 
through blood occlusion by external force. Chen of CIOMP attempted to reduce the background interference using subtracted blood volume spectrometry. ${ }^{17}$ Healthstat Company of Singapore intended to apply long wavelength laser to measure blood glucose by finger transmission spectroscopy. ${ }^{18}$ GlucoLight Company of America focused on the identification of special anatomic area on the skin that was sensitive to the blood glucose. ${ }^{19}$

Our group has also emphasized on the innovation of noninvasive blood glucose detection methods using NIR spectroscopy, and initially proposed the concepts of "float reference point" 20,21 and "aplanatic point" 22 to meet the challenges of weak signal, significant sensitivity to the individual difference and large body background and instrument shift. We find that, in the aqueous glucose solution (pure absorption medium), the absorbance does not change with the glucose concentration at certain wavelengths, which is expressed by

$$
\left.\frac{d A}{d C_{g}}\right|_{\lambda=\lambda_{r}}=0
$$

Therefore, the absorbance values at these wavelengths can reflect all the interferences which can be regarded as "reference" to remove the interferences from the spectra. Such wavelengths are named "reference wavelengths" and their existence is explained by the replacement between water and glucose molecules. ${ }^{23}$

Similar "references" are also proved in the intralipid glucose solution (scattering medium, phantom of human body) by further studies including Monte Carlo simulation and phantom experiments, and named "floating references" since they dynamically represent interferences, and their existence properties are influenced by the source-detector separation and wavelengths. ${ }^{24}$ We have also tried to explain their existence that both absorption and the change in the scattering light with the concentration of interesting component, and the "float references" appear-when the balance between the two effects is reached, which can be given by

$$
\frac{d \Phi}{d c_{g}}=\frac{\partial \Phi}{\partial \mu_{a}} \cdot \frac{\partial \mu_{a}}{\partial c_{g}}+\frac{\partial \Phi}{\partial \mu_{s}^{\prime}} \cdot \frac{\partial \mu_{s}^{\prime}}{\partial c_{g}}=0
$$

Moreover, the novel idea of "float reference" can also be applied to improve temperature measurement of certain solutions. ${ }^{25}$
However, though new theories and methods bloom in the field of noninvasive detection of body composition since 1970s, none of them have achieved clinical precision because of other major interference factors.

\subsection{Measurement conditions}

Online monitoring using NIR spectroscopy can be easily achieved in the fields of food safety and environment monitoring, because the measurement conditions are simpler.

However, such measurement conditions cannot meet the challenges of noninvasive detection of body composition. The noninvasive detection of body composition using NIR spectroscopy is an indirect method based on complex models. Taking noninvasive blood glucose detection as an example, the blood glucose is predicted based on the model of photon transmission in the tissue. The diffuse equation is one of the basic models that express such interactions. However, it can only be solved under certain restrictions, for example, one of which to achieve analytical solution to the stationary diffuse equation in infinite medium is that the reduced scattering coefficient $\left(\mu_{s}^{\prime}\right)$ should be much larger than the absorptive coefficient $\left(\mu_{a}\right)$. Such condition cannot be fully satisfied at wavelengths longer than $1500 \mathrm{~nm}$. However, such wavelength range is just where the glucose absorptive characteristics exist. The Monte Carlo method is also believed to be the most precise model to simulate the interactions between the photons and the tissue. However, the human skin is usually treated as ideal scattering medium with three individual layers (the epidermis, dermis and subcutaneous tissue) that contain homogeneous absorptive and scattering coefficients, respectively. Meanwhile, no interference factors, such as background shift or random noise are added during the simulation, which is quite different from the real situations. ${ }^{26}$ On the one hand, the calculation is much more ideal and convenient due to the modeling methods; on the other hand, the measurement conditions become much restricted and difficult to achieve.

Besides, the measurement precision is also influenced by the position selection and repeatability, contact pressure and temperature. ${ }^{27,28}$ In the noninvasive blood glucose detection, positions that are 
exposed and convenient to measure are the priorities for the patients, followed by other advantages, like blood abundance, less interferences by other components, individual difference or environment. Therefore, palm, forearm, finger and earlobe usually catch the attentions. ${ }^{29-32}$ The position repeatability demands precise adjustment of mechanical parts, and according to Kudo, repeatability can be greatly improved if the location error is less than $0.2 \mathrm{~mm} .{ }^{33}$ We also find that the contact pressure and the temperature can significantly influence the NIR diffuse-reflectance spectra, and according to Rong Liu, the diffuse-reflectance light abated when the contact pressure increased at wavelength range of 1100-1700 nm. ${ }^{34}$ Also Chenxi studied the influence of contact pressure on the NIR diffuse-reflectance spectra measurement. Based on the solid-liquid two-phase model, he also explored the tissue compressive deformation by measuring bound water and free water concentrations by second-order derivative pressure modulation diffuse-reflectance spectrophotometry. ${ }^{35}$ Meanwhile, Chen analyzed the influence of the temperature on the NIR diffusereflectance spectra measurement. ${ }^{23}$

The rigorous requirements of measurement conditions raise the level of instrument precision, increase difficulties and cost of instrument design. Those interferences induced by the measurement conditions should be removed by precise control, model compensation and reference measurement method.

\subsection{Instrument precision}

The noninvasive detection of body composition, compared with other fields of detection using NIR spectroscopy, such as food safety and environment monitoring, requires enhancement of the instruments precision with higher signal to noise ratio (SNR) and stability because of the weak signal, large background shift and rigorous measurement conditions.

Our group also focused attention on the development of NIR spectrometer with satisfying SNR and stability for noninvasive blood glucose detection, and made several generations of prototypes. $^{36,37}$ The spectrometer consists of a halogen tungsten lamp, an acousto-optic tunable filter (AOTF), and single-wavelength is diffracted by the AOTF using orthogonal polarization method. The halogen tungsten lamp with stable illumination and long-life endurance was surrounded by a three-layer chamber to remain with high intensity and stability. AOTF, with the advantages of nonmoving mechanical parts, programmable control and fast switch of wavelengths, makes it possible for the NIR online detection. The orthogonal polarization method can greatly reduce the background light, which on the contrary, highlights the useful optical signal. The spectrometer also includes photoelectric transducers with high conversion efficiency, sensitivity to the NIR wavelengths, low dark current and shot noise, which improves the SNR of the system. In the fiber optics probe design, maximum intensity of useful signal is achieved from both simulation and experiments through balancing the area of the incident and receiving rings. ${ }^{20}$

Furthermore, novel probe structure that is more sensitive to the diffuse-reflectance photons propagating through dermis is designed. ${ }^{38}$ For the pretreat methods, average filtering is adopted to reduce the random noises, and dual optical path is applied to remove interferences such as background and instrument shifts. ${ }^{36,37}$ The spectrometer can finally achieve a satisfying short-time SNR of 5000:1 and a long-time SNR of 10,000:1, and the stability represented by a variable coefficient (CV) of about $0.01 \%$ when samples are repeatedly measured.

The advancement of the NIR spectrometer depends largely on the novel design of the optical system and suitable signal processing and modeling methods, on which sufficient attention should be paid in order to meet the challenge of NIR noninvasive detection of body composition.

\subsection{Multi-component influence}

The human body is commonly defined as complex scattering medium in the biomedical photonics. Taking noninvasive blood glucose detection as example, the blood consists of $90-91 \%$ water, $6.5-$ $8.5 \%$ proteins and $2 \%$ metabolites and other hormones. The absorption bands usually result from the overtone and combination of the $\mathrm{C}-\mathrm{H}, \mathrm{O}-\mathrm{H}$ and $\mathrm{N}-\mathrm{H}$ vibrations in the NIR. Therefore, some absorption bands of haemoglobin, albumin, water and glucose are overlapped, which influences the measurement of interesting component.

We find that, the chance correlation among the concentrations in a multi-component medium 


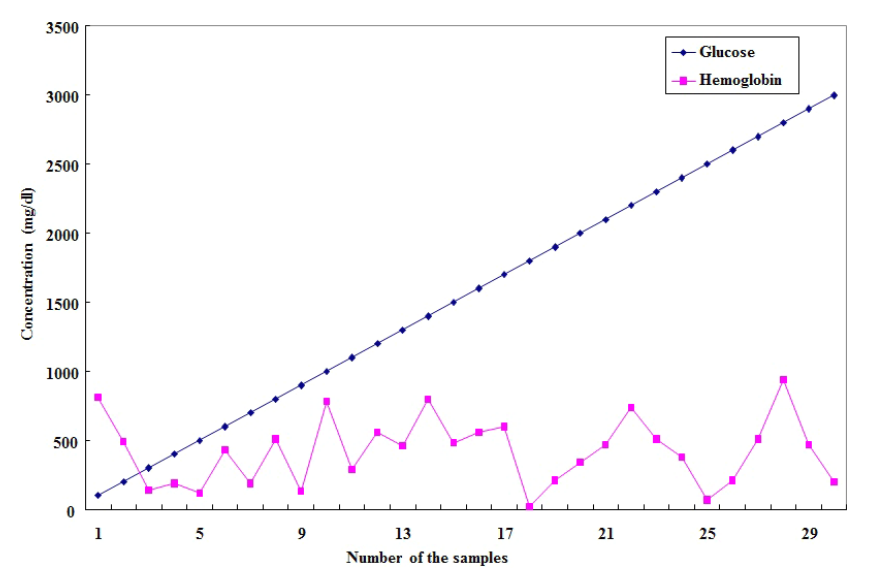

(a)

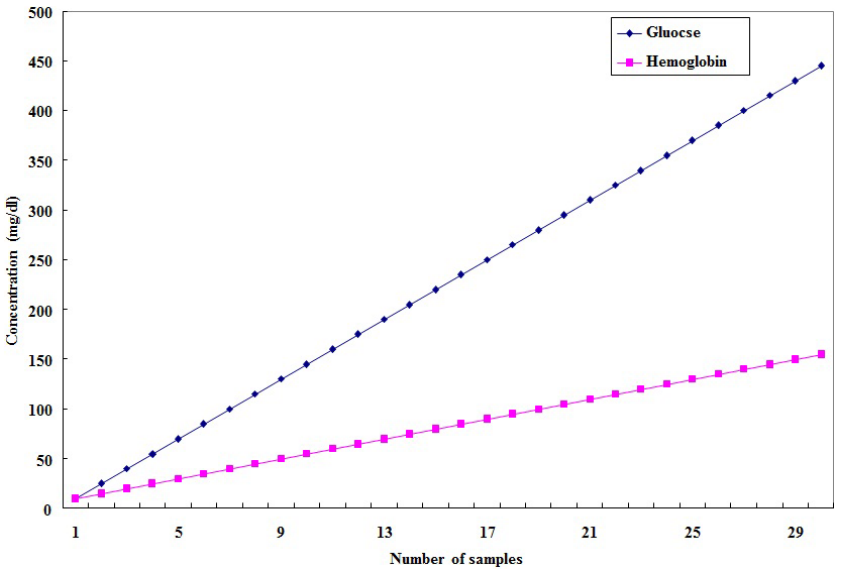

(b)

Fig. 2. Comparison between (a) uncorrelation between concentrations of glucose and hemoglobin and (b) chance correlation between concentrations of glucose and hemoglobin.

deserves attentions. ${ }^{39,40}$ As illustrated in Fig. 2, when the concentration of an interfering component happens to have a similar change as that of the interesting component, chance correlation occurs and the multivariate regression model will take both changes as useful signal induced by the glucose. However, when the "chance correlation" disappears later, the previous model will be out of predictability. The reason is that the multivariate regression model emphasizes on the variance of the spectrum induced by all the components. ${ }^{41}$ Furthermore, chance correlations can also result from instrument shift and temperature, which decreases the prediction precision. ${ }^{39,40}$

To solve the problem, we propose that the random sampling is the best way to avoid the chance correlation induced by the instrument shift, while in the in vitro multi-component experiments, chance correlation induced by the interference components should be averted by suitable design of the steps. As to the in vivo experiments, such chance correlations cannot easily be removed since measurement conditions and physiological status cannot be intentionally controlled. Chance correlation is one of the challenges confronting the selection of modeling method in the noninvasive body composition detection, and our group also tries to lucubrate from the aspect of net signal extraction. ${ }^{42}$

In short, in the multi-component medium, overlap of the absorption bands among different components, chance correlations induced by interference components and instrument shift require innovation of modeling methods and suitable experiment design.

\subsection{Individual difference}

Individual difference is one of the major challenges that specially define the applicability of methods on the objectives. For example, the individual difference means different tissue optical properties (absorption coefficient, scattering coefficient, refractive index, anisotropic factor, etc.) in the noninvasive blood glucose detection. The variations of a certain parameter will bring changes in the spectra just as the concentration change of interesting component. Therefore, the concentration of the interesting component cannot be distinguished under obvious individual difference.

We carry out several relevant studies to solve the problem. First, in order to study the influence of individual difference on the NIR spectrum measurement, we have defined, both by Monte Carlo and phantom experiments, that $3 \%$ intralipid has the maximum probable parameters as human tissue. Conclusions made on such basis can be more confidently applied to the in vitro and in vivo experiments, such as the floating reference method. ${ }^{23,37-43}$

Moreover, we attempt to develop new methods of reconstructing the parameters based on the diffusereflectance spectroscopy. ${ }^{44}$ Chenxi Li put forward a novel parameter reconstruction method in the following steps, as shown in Fig. 3. First, diffuse-reflectance spectra is calculated by Monte Carlo simulation with defined parameters and simplified by principal component analysis (PCA). Then, a neural network is trained by nonlinear regression between the parameters and the scoring matrix of the spectra. 
W. Chen et al.

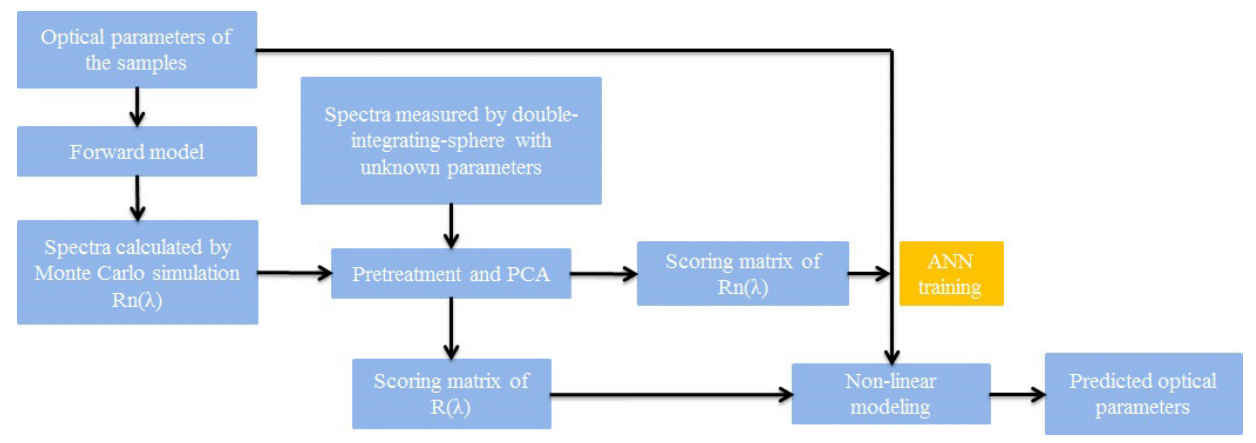

Fig. 3. Schematic of novel parameter reconstruction method using PCA + ANN.

Meanwhile, diffuse-reflectance spectra of samples with unknown parameters are measured by a doubleintegrating-sphere system and also simplified by PCA. Finally, the unknown parameters can be reconstructed when the scoring matrix of the measured spectra are put into the trained neural network. The development of novel parameter reconstruction method, with advantages of broad applicability and flexibility, has its practical value when individual difference exists, which puts the theories proved by phantom, in vitro and in vivo experiments into practice.

\subsection{Novel weak-signal extraction method}

After all, the noninvasive detection of body composition is still a kind of weak signal measurement which not only depends on the high SNR and stability of the instrument, but also calls for a novel weak-signal extraction method from complex inferences. The reference measurement method has been proved effective for background subtraction. As above mentioned, our group has developed a novel "floating reference method" to extract the weak glucose signal in the NIR spectra, ${ }^{23,36,37}$ as shown in Fig. 4.

The idea of this method is that, in the noninvasive blood glucose detection, the change in the diffusereflectance light includes useful signal induced by the glucose change as well as interferences, such as background and instrument shift. However, the diffuse-reflectance light does not change with the glucose concentration at certain source-detector separations or wavelengths, and thus can be regarded as "float reference" to remove all the interference
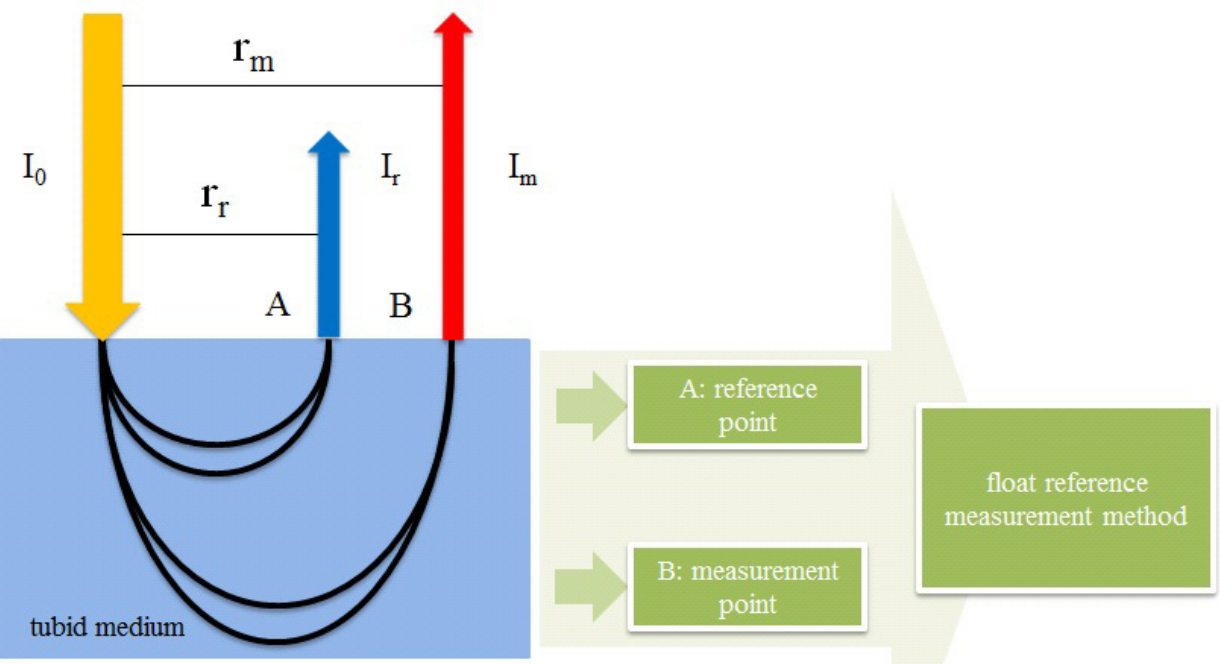

Fig. 4. Principles of float reference measurement method. 
factors. Since the fiber optics probe is designed with a detecting ring $(\mathrm{B})$ and a reference ring $(\mathrm{A})$, the NIR spectra collected by the detecting ring can be corrected based on those of the reference ring. The results indicate that, the float reference method can greatly reduce the calibration and prediction error of the partial least square (PLS) regression model. ${ }^{43}$

However, the float reference method also has its limitations. ${ }^{21,46}$ These references are sensitive to the tissue parameters, such as absorption coefficient and scattering coefficient, i.e., the method is sensitive to individual difference which, on the other hand, restricts the design of the fiber-optics probe. Adjustable reference ring will surely increase the difficulties and the cost of the probe design. To solve the problem, we have tried to adopt eight individual detecting optic fibers which are distributed in a ring, and movable by their respective stepping motors. ${ }^{47}$ We also focus our attentions on developing the float reference method at multi-wavelengths, and broad our vision from 1-dimensional spectrum to 2-dimensional or 3-dimensional hyper spectral imaging. Such innovation attempts may bring more abundant useful information for the detection of interesting components. In all, compared with advancement in instrument precision, novel weaksignal extraction method plays a key role in dealing with the challenges in the noninvasive detection of body composition using NIR spectroscopy.

\section{Conclusion}

The noninvasive detection of body composition, compared with other fields of detection using NIR spectroscopy, such as food safety and environment monitoring, requires higher precision of the instruments as well as more rigorousness of measurement conditions. Therefore, new challenges emerge when the NIR spectroscopy is applied to the noninvasive detection of body composition.

In this paper, based on our previous research in the cutting-edge field of NIR noninvasive blood glucose detection, new challenges in the noninvasive detection of body composition using NIR spectroscopy are concluded from aspects of measurement methods, measurement conditions, instrument precision, multi-component influence, individual difference and novel weak-signal extraction method. Moreover, novel ideas and approaches proposed by our group to solve the problems are introduced.
With the development of NIR noninvasive detection of body composition, technique innovations will play a significant role in meeting the new challenges, which includes new principles and theories, advanced instrument designs, and effective interference and individual difference reduction and weak-signal extraction methods. Those innovations require not only the extension of current methods but also the complete exploration of new areas. Since the NIR noninvasive detection of body composition is a cross-disciplinary subject, the innovations also depend greatly on the new theories and methods in mathematics and physics, and experience from other areas related to the noninvasive detection, such as magnetic resonance imaging and diffuse-reflection tomography.

\section{Acknowledgments}

Wenliang Chen, Hao Jia, Chenxi Li and Kexin Xu contributed equally to this work.

\section{References}

1. S. Simon, H. Andrew, "Diabetes and obesity: The twin epidemics," Nat. Med. 12, 75 (2006).

2. W. Yang, J. Lu, J. Weng, W. Jia, L. Ji, J. Xiao et al., "Prevalence of diabetes among men and women in China," N. Engl. J. Med. 362, 1090-1101 (2010).

3. C. E. Ferrante do Amaral, B. Wolf, "Current development in non-invasive glucose monitoring," Med. Eng. Phys. 30(5), 541-549 (2008).

4. K. Maruo, M. Tsurugi, M. Tamura et al., "In vivo noninvasive measurement of blood glucose by nearinfrared diffuse-reflectance spectroscopy," Appl. Spectrosc. 57(10), 1236-1244 (2003).

5. K. X. Xu, Q. B. Li, Z. L et al., "Fundamental study on non-invasive blood glucose sensing," J. X-Ray Sci. Tech. 10(3-4), 187-197 (2002).

6. G. C. Marten, J. L. Halgerson, J. H. Cherney, "Quality prediction of small grain forages by near infrared reflectance spectroscopy," Crop Sci. 23(1), 94-96 (1983).

7. Z. H. He, W. L. Lian, M. J. Wu et al., "Determination of tobacco constituents with acousto-optic tunable filter-near infrared spectroscopy," J. Near Infrared Spectrosc. 14(1), 45-50 (2006).

8. B. M. Nicolaï, K. Beullens, E. Bobelyn et al., "Nondestructive measurement of fruit and vegetable quality by means of NIR spectroscopy: A review," Postharvest Biol. Technol. 46(2), 99-118 (2007). 
9. D. B. Zou, W. L. Chen, Z. H. Du et al., "Selection of digital filtering in the escaping ammonia monitoring with TDLAS," Spectrosc. Spectral Anal. 32(9), 2322-2326 (2012).

10. M. A. Arnold, G. W. Small, "Noninvasive glucose sensing," Anal. Chem. 77(17), 5429-5439 (2005).

11. N. Kaiser, "Method for determining the contents of metabolic products in the blood," US Patent 4169676 (1979).

12. F. Jobsis, "Noninvasive infrared monitoring of cerebral and myocardial oxygen sufficiency and circulatory parameters," Science 198(4323), 1264-1267 (1977).

13. C. Dahne et al., "Spectrophotometric method and apparatus for the non-invasive determination of glucose in body tissues," US Patent 4655225 (1987).

14. A. J. Berger et al., "Rapid non-invasive concentration measurements of aqueous biological analytes by near infrared Raman spectroscopy," Appl. Opt. 77(1), 13-19 (1981).

15. K. V. Larin et al., "Noninvasive blood glucose monitoring with oct technique: A pilot clinical study in human subjects," Diabetes Care 25(12), 22632267 (2002).

16. O. Cho et al., "Noninvasive measurement of glucose by metabolic heat conformation method," Clin. Chem. 50, 1894 (2004).

17. X. D. Chen, "Possibility of noninvasive clinical biochemical examination by near infrared spectroscopy," Opt. Precision Eng. 16(5), 759-763 (2008).

18. http://www.healthstats.com/en/executive-team. html.

19. http://www.diabeteshealth.com/read/2007/04/17/ 5124.html.

20. Y. Yang, Z. Z. Shi et al., "Simulation and validation of the radial reference point in non-invasive blood glucose sensing by NIR," Nanotechnol. Precision Eng. 8(2), 114-119 (2010).

21. Y. Yang, K. Xu et al., "Influence of skin tissue properties on the radial reference point for glucose measurement," Proc. SPIE, Vol. 7186, 71860N (2009).

22. Z. Du, C. Li et al., "Research on the spectroscopy of turbid media with optical-length resolved detection," Proc. SPIE, Vol. 7572, 75720H (2010).

23. Y. Chen, W. Chen, Z. Shi, Y. Yang, K. Xu, "A reference-wavelength based method for improved analysis of near-infrared spectroscopy," Appl. Spectrosc. 63(5), 544-548 (2009).

24. Y. Yang, W. Chen et al., "Reference point of floating-reference method for blood glucose sensing," Chin. Opt. Lett. 8(4), 421-424 (2010).

25. Y. Chen, Y. J. Liang, W. L. Chen, K. X. Xu, "Measurement of solution temperature using reference-wavelength method," Spectrosc. Spectral Anal. 29(10) 2657-2660 (2009).
26. R. Liu, B. Deng, W. Chen, K. Xu, "Next step of noninvasive glucose monitor by NIR technique from the well-controlled measuring condition and results," Opt. Quantum Electron. 37, 1305-1317 (2005).

27. W. Chen, R. Liu, K. Xu, "Influence of contact state on NIR diffuse reflectance spectroscopy in vivo," J. Phys. D Appl. Phys. 38(15), 2691-2695 (2005).

28. K. $\mathrm{Xu}$ et al., "Non-invasive glucose sensing with near-infrared spectroscopy enhanced by optical measurement conditions reproduction technique," Opt. Lasers Eng. 43(10), 1096-1106 (2005).

29. K. Jagemann, C. Fischbacher, K. Danzer et al., "Application of near-infrared spectroscopy for noninvasive determination of blood/tissue glucose using neural networks," Zeitschrif. für Physikalische Chem. 191, 179-190 (1995).

30. M. R. Robinson, R. P. Eaton, D. M. Haaland et al., "Noninvasive glucose monitoring in diabetic patients: A preliminary evaluation," Clin. Chem. 38, 1618-1622 (1992).

31. B. D. Cameron, H. W. Gorde, B. Satheesan et al., "The use of polarized laser light through the eye for noninvasive glucose monitoring," Diabetes Technol. Ther. 1(2), 135-143 (1999).

32. P. G. Steffes, "Laser-based measurement of glucose in the ocular aqueous humor: An efficacious portal for determination of serum glucose levels," Diabetes Technol. Ther. 1, 129-133 1999.

33. H. Kubo, Y. Mitsumura, H. Uenoyama et al., "Method and apparatus for measuring concentration by light projection," US Patent, 6147749 (2000).

34. R. Liu, K. Xu, et al., "Combined optimal-pathlengths method for near-infrared spectroscopy analysis," Phys. Med. Biol. 49, 1217 (2004).

35. L. Chenxi, Z. Huijuan, S. Zhenzhi, X. Kexin, "Effect on glucose monitoring of pressure exerted by fiberoptic probe: Skin model and simulation," Proc. SPIE, Vol. 8229, 82291H (2012).

36. Z. Shi, Y. Yang, H. Zhao, W. Chen, R. Liu, K. Xu, "Theoretical studies of floating-reference method for NIR blood glucose sensing," Optical Diagnostics and Sensing XI: Toward Point-of-Care Diagnostics and Design Land Performance Validation of Phantoms Used in Conjunction with Optical Measurement of Tissue III, Proc. SPIE, Vol. 7906, 79060W-9 (2011).

37. J. Zheng, K. Xu, Y. Yang, "An effective method based on reference point for glucose sensing at 11001600 nm," Optical Diagnostics and Sensing XI: Toward Point-of-Care Diagnostics; and Design and Performance Validation of Phantoms Used in Conjunction with Optical Measurement of Tissue III Proc. SPIE, Vol. 7906, 7906A-16 (2011).

38. L. Chenxi, W. Qiuyin, S. Zhenzhi, L. Yunhan, Z. Huijuan, X. Kexin, "Analysis and modification of 
estimate error in optical properties measurement with the double-integrating-spheres system," Proc. SPIE, Vol. 7563, 75630O-9 (2010).

39. R. Liu, W. Chen, X. Gu, K. Xu, "Chance correlation in the non-invasive glucose measurement using nearinfrared spectroscopy," J. Phys. D Appl. Phys. 38(15) 2675-2681 (2005).

40. W. J. Zhang, R. Liu, W. Zhang et al., "Discussion on the validity of NIR spectral data in non-invasive blood glucose sensing," Biomed. Opt. Express 4(6), 789-802 (2013).

41. F. Vogt, K. Booksh, "Influence of wavelengthshifted calibration spectra on multivariate calibration models," Appl. Spectrosc. 58, 624-635 (2004).

42. E. B. Carlos, A. C. Olivieri, "Net analyte preprocessing: A new and versatile multivariate calibration technique: Analysis of mixtures of rubber antioxidants by NIR spectroscopy," J. Near Infrared Spectrosc. 9, 245-254 (2001).

43. R. Liu, B. He, K. Xu, Y. Yang, "Data correction method based on the floating reference point for the noninvasive glucose sensing," Optical Diagnostics and Sensing IX Proc. SPIE, Vol. 7186, 71860O-9 (2009).

44. L. Chenxi, Z. Huijuan, M. Jierong, L. Julan, X. Kexin, "Improved double-integrating-spheres system for multiwavelength optical properties measurement: Investigation and application," Proc SPIE, Vol. 7176, 71760J (2009).

45. L. Chenxi, W. Qiuyin, Z. Huijuan, X. Kexin, "Artificial neural network method for determining optical properties from double-integrating-spheres measurements," Chin. Opt. Lett. 02, 173-176 (2010).

46. R. Liu, W. Chen, Y. Yang, Y. Li, K. Xu, "The wavelength-dependent characteristic of the floatingreference point in the non-invasive glucose sensing," Proc. SPIE, Optical Diagnostics and Sensing VIII, Vol. 6863 (2008).

47. J. Jiang, X. Rong, H. Zhang, K. Xu, "A device to improve the SNR of the measurement of the positional floating reference point," Proc. SPIE, Dynamics and Fluctuations in Biomedical Photonics IX, Vol. 8580 (2013). 